Interação Psicol.,15(1), 11-19

\title{
Percepção, Atratividade e Probabilidade de Engajamento em Situações de Risco: Diferenças de Gênero
}

\author{
Eulina da Rocha Lordelo*, Aline Ferreira Campos, Carla Silva Fiaes, Rachel Coelho Ripardo, \\ Samai Alcira Cunha, \& Sâmia de Carliris Oliveira Barbosa \\ Universidade Federal da Bahia, Salvador, Brasil
}

\begin{abstract}
RESUMO
Diferenças de gênero em comportamentos de risco podem ser explicadas pela teoria evolucionista, que prediz diferenças conforme o domínio avaliado. Visando a investigar esse problema, utilizou-se uma escala de propensão ao risco com 358 pessoas, que responderam um questionário de 15 itens, cobrindo cinco domínios, avaliados quanto às dimensões Percepção, Atratividade e Probabilidade de engajamento. Em geral, foram encontradas diferenças de gênero nas dimensões, mas a análise dos domínios sugeriu diferenças apenas em Desafio ambiental, e Acasalamento e recursos. Esses resultados confirmam a literatura quanto à maior propensão dos homens ao risco, mas também sugerem que o tipo de domínio envolvido pode ser relevante na propensão ao risco. Discute-se a necessidade de novas pesquisas que refinem os presentes achados.
\end{abstract}

Palavras-chave: percepção de risco; comportamento de risco; psicologia evolucionista; diferenças de gênero.

\section{ABSTRACT \\ Perception, Attractiveness and Likelihood of Engaging in Situations of Risk: Gender Differences}

Gender differences in risk behaviors can be explained by evolutionary theory, which predicts differences in the domain under analysis. In order to investigate this question, we used a risk propensity scale with 358 people, who answered a questionnaire of 15 items covering five domains, evaluated concerning the dimensions Perception, Attractiveness and Probability of engagement. In general, gender differences were found in the dimensions, but the analysis of the domains suggested differences only in Environmental challenge, and Mating and resources. These results are consistent with the literature about the greater propensity of men to risk, but also suggest that the domain involved may be relevant in risk propensity. The need for further research to refine the present findings is discussed.

Keywords: risk perception; risk behavior; evolutionary psychology; gender differences.

Diferenças de gênero em comportamentos de risco vêm sendo encontradas em muitos estudos, mesmo aqueles não orientados pela perspectiva evolucionista. A pesquisa epidemiológica, especialmente, tem revelado diferenças consistentes entre os gêneros quanto à maior prevalência do abuso de bebidas alcoólicas, fumo e menor percepção de níveis de estresse e de autoavaliação negativa do estado de saúde entre ho- mens, enquanto entre as mulheres há uma maior prevalência de riscos relacionados à inatividade física e ao estresse (Barros \& Nahas, 2001; Colares, Franca, \& Gonzalez, 2009; Martins, Tavares, Lobo, Galetti \& Gentil, 2004).

Outro aspecto de grande preocupação e alvo de pesquisas na área consiste no comportamento sexual de risco associado ao uso de substâncias injetáveis,

\footnotetext{
Endereço para correspondência: Eulina da Rocha Lordel, Rua Ranulfo Oliveira, 1007, Apto. 202, Salvador, BA, CEP 40155-030. Email: eulinalordelo@gmail.com.
} 
que aumenta o risco de infecção por HIV e outras doenças sexualmente transmissíveis. Diversos estudos nacionais e internacionais apontam uma maior prevalência desses comportamentos no sexo masculino, que faz uso mais frequente de drogas ilícitas, de substâncias para auxiliar a ereção e de sexo sem proteção, além de possuir uma maior quantidade de parceiros sexuais (Colares e cols., 2009; Courtenay-Quirk e cols., 2008; Lejuez, Simmons, Aklin, Daughters, \& Dvir, 2004; Martins e cols., 2004; Silveira, Béria, Horta, \& Tomasi, 2002).

Ao lado dos estudos descritivos, alguns pesquisadores têm direcionado seus esforços para construir teorias que expliquem os motivos que levam as pessoas a comportarem-se de modos que ameaçam sua vida/saúde, incluindo teorias de cunho econômico; como as teorias do valor esperado e do risco-retorno; de cunho desenvolvimental; as quais relacionam as habilidades em fazer escolhas tendo como referência o aparato neurológico envolvido; bem como a partir de teorias cognitivas e de atitudes de risco, estas últimas tradicionais na psicologia social (Reyna \& Rivers, 2008; Weber, Blais, \& Betz, 2002).

De acordo com Weber \& cols. (2002), as expressões "atitude de risco", "aversão a risco" e "propensão a risco" são normalmente interpretadas como traços de personalidade, para os quais as pessoas variam num continuum. No entanto, essa visão tem sido desafiada por alguns resultados inconsistentes, apontando para diferenças relacionadas ao domínio em questão (Schoemaker, 1993), bem como para a instabilidade dos resultados conforme o contexto da investigação (Weber e cols., 2002) e, especialmente, conforme a dimensão investigada seja do risco percebido ou da atitude em relação à atividade percebida como arriscada.

Uma vez que muitas escalas utilizadas para medir atitudes de risco confundiam essas duas dimensões, Weber e cols. (2002) desenvolveram uma escala para avaliar diferenças individuais em torno de atitudes de risco. Essa escala traz duas contribuições em relação às demais, sendo a primeira delas a distinção de duas variáveis psicológicas que até então não eram consideradas de formas distintas, a saber, a percepção de risco e a atitude em torno do risco percebido. Além disso, a escala também examina a tomada de comportamentos de risco e seus determinantes em diferentes áreas de conteúdos, como a saúde/segurança, a ética, a social, os riscos recreacionais e os riscos financeiros este último subdividido em risco de investimento e jogo.

Um dos principais achados do estudo acima referido foi o grau de assunção de riscos, que era altamente variável conforme o domínio avaliado, ou seja, os indivíduos não eram "buscadores de risco" ou "avessos a riscos" de forma consistente em todos os domínios. Além disso, os resultados sugeriam que a probabilidade de a pessoa engajar-se em atividades arriscadas, que variava em função do gênero e do domínio, estava associada à percepção da relação "risco versus benefício", ao invés da percepção do risco em si.

Levando em consideração que estes resultados permitiam uma exploração sistemática das diferenças entre os domínios de conteúdo, mas não traziam razões teóricas subjacentes para definir quais domínios deviam ser escolhidos, Kruger, Wang e Wilke (2007) adotaram a perspectiva da psicologia evolucionista para identificar os domínios específicos de tomada de comportamentos de risco na construção de uma escala de propensão a assumir riscos.

A abordagem da psicologia evolucionista assume que os programas que compreendem a mente humana foram desenhados pela seleção natural para solucionar problemas recorrentes enfrentados pelos nossos ancestrais caçadores-coletores, tais como a escolha de parceiros, cooperação com outros, caça, coleta, proteção da prole, navegação, evitação de predadores, evitação de exploração, etc. (Tooby \& Cosmides, 1995).

Além de considerar que as adaptações exibidas atualmente foram selecionadas no passado evolutivo, a psicologia evolucionista considera que, naquele ambiente, foram selecionados módulos de domínio específico, que evoluíram em resposta a pressões específicas do ambiente (Tooby \& Cosmides, 1995; Yamamoto, 2009). Ou seja, como os problemas enfrentados pelos nossos ancestrais no ambiente de evolução adaptativa eram específicos, os módulos selecionados forneceriam informações específicas necessárias para resolver cada tipo de problema.

Por essa perspectiva, é possível predizer que a tomada de decisão e a propensão a assumir riscos devem ser diferenciadas conforme domínios específicos para os riscos evolutivamente relevantes, que podem ainda existir nos contextos contemporâneos. Assim, situações análogas às enfrentadas pelos nossos ancestrais tenderiam a ativar algoritmos construídos para 
resolver os tais problemas (Wang, Kruger, \& Wilke, 2009).

Desse modo, Kruger \& cols. (2007) levaram em consideração os diferentes tipos de desafios que nossos ancestrais enfrentaram durante a história evolucionista e delimitaram a existência de cinco domínios para a construção da escala de propensão a riscos, são eles: Desafio ambiental, Acasalamento e recursos, Competição intergrupal, Competição intragrupal, e Fertilidade e reprodução.

O domínio Desafio ambiental remete-se aos contextos já enfrentados pelos nossos ancestrais, e, assim, relacionam-se à procura por recursos, como por exemplo, através da caça, coleta e evitação e fuga de predadores e de ameaçadores ambientais da sobrevivência humana. Em seguida, o domínio Acasalamento e recursos refere-se ao investimento diferenciado de machos e fêmeas na procura por parceiros sexuais; homens, de modo geral, usam estratégias de curto prazo, procurando envolvimento com um grande número de parceiras, ao contrário das mulheres, que se utilizam de estratégias de longo prazo e um menor número de parceiros.

Já o domínio Competição intergrupal, diz respeito à necessidade de manter limites territoriais, tanto na mesma espécie, como com outras espécies. Pode-se identificar, como exemplo na atualidade, a tendência à formação da identidade social com base em comparações intergrupais.

Por outro lado, a Competição intragrupal possibilita a definição do status do sujeito no grupo social e envolve desde a luta física até outras formas de competição social. Como exemplo, podemos perceber a tendência de ocorrer, em um determinado grupo social, a maior valorização de membros bem-sucedidos, seja financeiramente, fisicamente, etc.

Um dos últimos domínios delimitados por Kruger $\&$ cols. (2007) consistiu no risco à fertilidade e à reprodução, no qual foi considerado que o objetivo final da evolução animal é a reprodução. Admitindo a existência desses diferentes domínios, seria então esperado que Percepção de risco, Atração e Probabilidade de engajamento em situações arriscadas, embora relacionados, constituam fenômenos relativamente diferentes, sob controle de variáveis diversas. Também seria esperado que homens e mulheres se diferenciassem quanto a essas dimensões.
De fato, os resultados de Kruger \& cols. (2007) para as diferenças de gênero apontaram que homens engajaram-se em comportamentos de risco mais frequentemente do que as mulheres nos cinco domínios analisados.

Portanto, diante da análise dos estudos sobre comportamentos de riscos associados à perspectiva evolucionista, torna-se relevante a realização de pesquisas em diferentes sociedades com o objetivo de informar como os domínios específicos de riscos variam de acordo com os contextos e com as diferenças de gênero.

Assim, o presente estudo visou a identificar diferenças de gênero que estão relacionados à propensão a assumir riscos no contexto brasileiro, onde pesquisas em psicologia evolucionista de condições ambientais ainda são escassas.

\section{MÉTODO}

\section{Participantes}

Participaram da pesquisa 358 voluntários, com idade mínima de 18 anos e escolaridade de, pelo menos, ensino médio completo, pré-requisito para a participação. Tendo em vista o maior interesse da pesquisa em processos e mecanismos psicológicos, e não em estabelecer incidência dos fenômenos em investigação, não houve a preocupação em se estabelecer uma amostra representativa. A captação dos voluntários foi realizada de forma acidental, em diversos locais da região metropolitana de Salvador, e em Alagoinhas, cidade de cerca de 120 mil habitantes, no interior da Bahia.

\section{Materiais e procedimento}

O questionário utilizado no presente estudo foi traduzido e adaptado da Escala de Wang \& cols. (2009), a Risk-propensity Scale. Os itens da escala referem-se a cinco domínios de risco, resultantes de análise fatorial confirmatória e exploratória da proposta original de Kruger \& cols., (2007), a saber: (1) Desafio ambiental, (2) Acasalamento e recursos, (3) Competição intragrupal, (4) Competição intergrupal e (5) Fertilidade e reprodução. Para o presente estudo, pequenas alterações nas situações apresentadas foram realizadas, visando a tornar os itens mais condizentes com a realidade do contexto brasileiro. 
Em dois dos 15 itens, as situações apresentadas foram consideradas impróprias para o contexto brasileiro e ajustadas com situações supostamente equivalentes. Os itens "Dirigir até a universidade rival à noite e roubar a bandeira da escola hasteada no centro do campus" e "Perseguir um urso na área do seu acampamento batendo vasos e panelas" foram substituídos por "Dirigir de noite até onde está pendurada a propaganda política de um candidato do partido rival, e destruí-la" e "Durante um passeio em uma fazenda, espantar com uma vareta um touro bravo que se aproxima de seu grupo de forma ameaçadora".

A tradução e as adaptações foram avaliadas por um grupo de dez estudantes de pós-graduação, em duas etapas, visando a atingir a máxima clareza dos itens; as primeiras aplicações mostraram que todos os itens eram compreensíveis e não geravam desconforto nos respondentes, de modo que todos os participantes foram incluídos na amostra total.

No questionário os participantes deveriam avaliar esses 15 itens de comportamentos usando: a) uma escala bipolar de três pontos, em termos do grau de risco do comportamento (Nada arriscado, Moderadamente arriscado e Extremamente arriscado); b) uma escala bipolar de cinco pontos em termos de dimensões de atratividade pelo comportamento (Nada atraído, Pouco atraído, Não tenho certeza, Atraído e Muito atraído); c) por fim, uma escala bipolar de cinco pontos em termos de probabilidade de envolver-se na situação (Muito improvável, Pouco provável, Não tenho certeza, Provável e Muito provável). Os itens referentes aos cinco domínios foram distribuídos aleatoriamente ao longo do questionário.

Os participantes também forneceram informações sobre sete variáveis independentes de sua história de vida: sexo, idade, status conjugal, status parental, objetivo reprodutivo (número mínimo e máximo de fi- lhos desejados), número de irmãos, ordem de nascimento e expectativa subjetiva de vida.

A aplicação dos questionários se deu em ambientes reservados, estando o pesquisador disponível para esclarecimentos, mas fora do alcance das respostas dadas, de modo a garantir a privacidade dos participantes. Os questionários autorrespondidos (não identificados) e termos de consentimento assinados foram depositados, na presença do respondente, em envelopes separados.

Para o presente artigo, apenas a variável independente gênero foi analisada. A pesquisa foi submetida ao Comitê de Ética da Universidade Federal da Bahia e os participantes assinaram o Termo de consentimento livre e esclarecido.

\section{Análise dos dados}

As respostas aos itens foram submetidas aos procedimentos para obtenção de escores totais e das subescalas (soma dos itens de cada subescala, dividida pelo número de itens). Esses escores foram tratados como variáveis dependentes, para comparação de grupos, através do teste $\mathrm{t}$ ( $t$-test), tendo sido previamente conduzidos procedimentos para determinar a normalidade estatística das variáveis e a homogeneidade de variância entre os grupos.

\section{RESULTADOS}

A Tabela 1 apresenta as características sociodemográficas dos participantes do estudo. A idade variou entre 18 e 62 anos, com média de 28,5 anos (DP = 10). A análise descritiva dos dados indicou ainda que a maioria dos participantes do estudo era composta por solteiros e era do sexo feminino. A maioria não possuía irmãos ou possuía apenas um, sendo a maior parte dos participantes composta por primogênitos. 
Tabela 1

Frequências das Categorias Relativas aos Dados Sociodemográficos dos Participantes

\begin{tabular}{lc}
\hline Estado civil & Percentagem \\
\hline Solteiro & $74,0 \%$ \\
$\quad$ Casado/União consensual & $26,0 \%$ \\
Idade & \\
18 a 22 anos & $36,9 \%$ \\
23 a 30 anos & $32,1 \%$ \\
31 anos ou mais & $31,0 \%$ \\
Gênero & \\
Masculino & $37,9 \%$ \\
Feminino & $62,1 \%$ \\
Ordem de nascimento & \\
Primeiro & $37,7 \%$ \\
Segundo & $31,6 \%$ \\
Terceiro ou mais & $30,7 \%$ \\
Número de irmãos & \\
Nenhum ou um & $34,4 \%$ \\
Dois & $31,6 \%$ \\
Três ou mais & $34,1 \%$ \\
\hline
\end{tabular}

A Tabela 2, por sua vez, apresenta as médias de escores e desvios padrão nas dimensões Percepção de risco, Atratividade e Probabilidade de engajamento em situações de risco para homens e mulheres separadamente. Conforme pode ser visto na tabela, todas as três dimensões avaliadas foram afetadas pela variável gênero, com diferenças estatisticamente significativas. Assim, para a dimensão Percepção de risco, as mulhe- res apresentam escores médios mais altos do que os homens $(t=-2,327, \mathrm{p}<0,05)$.

Para a dimensão Atratividade, os homens apresentaram escores médios mais altos do que as mulheres $(t=2,961, \mathrm{p}<0,01)$. Do mesmo modo, para a dimensão de Probabilidade de engajamento, os escores médios dos homens foram mais altos do que os das mulheres $(t=3,948, \mathrm{p}<0,001)$.

Tabela 2

Médias, Desvio Padrão e Valor de t nas Dimensões Percepção de Risco, Atratividade ao Comportamento de Risco e Probabilidade de Comportamento de Risco, para Homens e Mulheres

\begin{tabular}{lcc}
\hline & $\begin{array}{c}\text { Homens } \\
(\mathrm{N}=135)\end{array}$ & $\begin{array}{c}\text { Mulheres } \\
(\mathrm{N}=221)\end{array}$ \\
\hline Percepção de risco & $32,27(3,39)$ & $36,09(2,92)$ \\
t-teste & & $-2,327^{*}$ \\
Atratividade ao comportamento de risco & $32,40(6,82)$ & $30,27(6,1)$ \\
t-teste & & $2,961^{* *}$ \\
Probabilidade do comportamento de risco & $32,52(6,3)$ & $30,0(5,4)$ \\
t-teste & & $3,948^{* * *}$ \\
\hline
\end{tabular}

Nota. ${ }^{*} p<0,05 .{ }^{* *} p<0,01 .{ }^{* * *} p<0,001$.

Conforme pode ser visto na Tabela 3, os domínios receberam avaliações diferentes para a percepção, sendo as ameaças à Fertilidade e reprodução o domínio com escore mais alto (7,9 para homens e 8,1 para mulheres), enquanto a Competição intragrupal foi 
vista como o domínio menos perigoso (5,6 para os dois gêneros).

A mesma tendência foi observada quanto à atratividade e a probabilidade de engajamento - aqueles domínios em que as ameaças são percebidas como menos perigosas, por exemplo, o domínio Competição Intragrupal desperta também mais atração $(9,5$ para os homens e 9,4 para as mulheres) e maiores chances de engajamento (10,2 para os homens e 10,0 para as mulheres).

\begin{tabular}{|c|c|c|c|}
\hline Domínios & $\begin{array}{l}\text { Homem } \\
\mathrm{M}(d p)\end{array}$ & $\begin{array}{l}\text { Mulher } \\
\text { M (dp) }\end{array}$ & t-teste \\
\hline \multicolumn{4}{|l|}{ Percepção de risco } \\
\hline Desafio ambiental & $7,0(1,1)$ & $7,3(1,0)$ & $-2,042^{*}$ \\
\hline Acasalamento e recursos & $7,7(1,2)$ & $7,9(0,9)$ & $-1,845$ \\
\hline Competição intragrupal & $5,6(1,2)$ & $5,6(1,1)$ & $-0,395$ \\
\hline Competição intergrupal & $7,1(1,2)$ & $7,2(1,1)$ & $-1,425$ \\
\hline Fertilidade e reprodução & $7,9(1,0)$ & $8,1(1,1)$ & $-1,103$ \\
\hline \multicolumn{4}{|l|}{ Atratividade } \\
\hline Desafio ambiental & $7,9(2,8)$ & $7,0(2,5)$ & $3,189^{* *}$ \\
\hline Acasalamento e recursos & $5,8(2,2)$ & $5,2(2,1)$ & $2,742^{* *}$ \\
\hline Competição intragrupal & $9,5(2,4)$ & $9,4(2,3)$ & 0,298 \\
\hline Competição intergrupal & $4,5(2,0)$ & $4,2(1,7)$ & 1,340 \\
\hline Fertilidade e reprodução & $4,8(2,0)$ & $4,6(2,0)$ & 0,909 \\
\hline \multicolumn{4}{|c|}{ Probabilidade de engajamento } \\
\hline Desafio ambiental & $7,9(2,9)$ & $6,8(2,3)$ & $3,710^{\star \star \star}$ \\
\hline Acasalamento e recursos & $5,5(2,1)$ & $4,7(1,8)$ & $3,521^{\star * *}$ \\
\hline Competição intragrupal & $10,2(2,3)$ & $10,0(2,2)$ & 0,977 \\
\hline Competição intergrupal & $4,3(1,6)$ & $4,0(1,6)$ & 1,365 \\
\hline Fertilidade e reprodução & $4,6(1,7)$ & $4,5(1,9)$ & 0,747 \\
\hline $\mathrm{N}$ & 135 & 221 & \\
\hline
\end{tabular}

Independentemente do domínio, os homens mostraram maior propensão ao risco em todas as dimensões, conforme visto acima, na Tabela 3. No entanto, quando os diferentes domínios são especificados, essas diferenças de gênero aparecem apenas em dois domínios: Desafio ambiental e Acasalamento e recursos. No primeiro caso, Desafio ambiental, ocorreram diferenças estatisticamente significativas em Percepção (7,0 para homens e 7,3 para mulheres), Atratividade (7,9 para homens e 7,0 para mulheres) e Probabilidade de engajamento (7,9 para homens e 6,8 para mulheres); no segundo, Acasalamento e recursos, a percepção é a mesma entre os sexos, mas Atratividade e a Probabilidade de engajamento são bem mais altos entre os homens (5,8 e 5,5, respectivamente) do que entre as mulheres $(5,2$ e 4,7$)$.

Em síntese, os homens sentem-se mais atraídos e engajados do que as mulheres em comportamentos que envolvem riscos relacionados ao Desafio ambiental e ao Acasalamento e recursos.

\section{DISCUSSÃO}

Conforme esperado e em acordo com a teoria evolucionista, os resultados do presente estudo mostraram que as três dimensões investigadas, Percepção de risco, Atratividade e Probabilidade de engajamento em situações de risco, apresentaram diferenças estatisti- 
camente significativas quanto ao gênero. Esses resultados sugerem que as mulheres têm uma maior percepção do risco, são menos atraídas e relatam uma menor probabilidade de engajamento em determinadas situações do que os homens. Ao contrário, os homens percebem as situações propostas como menos arriscadas, e apresentam uma maior atratividade e probabilidade de engajamento em comportamentos de risco.

Apesar de os nossos achados referirem-se a situações de risco hipotéticas, em que o indivíduo deve avaliar o quanto acha arriscada a situação, sente-se atraído e se envolveria nela, outros estudos que investigaram os relatos de indivíduos sobre suas condutas reais envolvendo riscos também encontraram diferenças importantes no envolvimento de homens e mulheres nessas situações (Colares \& cols, 2009; Garcia \& Costa Jr, 2008; Silveira \& cols, 2002).

Nesse sentido, os achados do presente estudo são compatíveis com os encontrados em várias outras pesquisas. Por exemplo, Colares e cols. (2009), ao investigar diferenças entre os gêneros nas condutas de saúde entre jovens universitários brasileiros, encontraram que as mulheres tinham frequências mais baixas do que os homens em condutas de risco relacionadas ao consumo de álcool, uso do tabaco e de drogas ilícitas, bem como de porte de arma e de envolvimento em briga física. Também foi encontrada maior ocorrência de comportamentos envolvendo riscos sexuais, como maior prática de atividade sexual na vida e uso de álcool e outras drogas antes da última relação sexual, entre jovens do sexo masculino do que do sexo feminino.

Do mesmo modo, Garcia e Costa Junior (2008) encontraram que meninos adolescentes escolares no México apresentavam valores significativamente mais altos de conduta antissocial e de consumo de álcool no último mês quando comparados ao grupo de estudantes do sexo feminino. O estudo de Silveira \& cols. (2002) também focalizou risco, mas apenas em mulheres. Nessa pesquisa, a autopercepção de risco não foi considerado um bom indicador do comportamento de risco, uma vez que muitas mulheres com um escore alto em comportamentos de risco não consideravam que tinham uma maior chance de contrair DST/AIDS. Esse resultado sugere que o comportamento de risco não pode ser predito apenas pela maneira como ele é percebido, embora as duas esferas possam estar relacionadas.

Os resultados do presente estudo também são concordantes com os achados do estudo original de Wang e cols. (2009) quanto às fortes diferenças entre homens e mulheres nas três dimensões. No entanto, quando se consideram os domínios específicos, notase a emergência de um quadro mais complexo. Especificamente, foram encontradas diferenças estatisticamente significativas envolvendo o gênero nas três dimensões investigadas quanto ao domínio Desafio ambiental e, nas dimensões Atratividade e Probabilidade de engajamento, quanto ao domínio Acasalamento e recursos. Esses resultados sugerem que as mulheres percebem mais do que os homens os riscos ambientais relacionados à manutenção da vida. Por outro lado, os homens atraem-se e engajam-se com mais frequência do que as mulheres em situações que envolvam riscos à manutenção da vida e em riscos relacionados à procura por parceiras sexuais.

Os achados também são concordantes com os encontrados no estudo de Weber e cols. (2002), que encontraram diferenças de gênero relacionadas à propensão para assumir riscos: os resultados desses autores foram interpretados como resultado da tendência masculina a um alto nível de esforço na procura de parceira e baixo nível de investimento parental, o que levaria a estratégias comportamentais mais arriscadas.

A teoria utilizada nesta pesquisa, assim como nos estudos de Wang e cols. (2009) fornece uma interpretação que pode ampliar a compreensão sobre o comportamento de risco. Achados como os de Colares e cols. (2009), Garcia e Costa Junior (2008), dentre outros, apontam para o maior envolvimento de homens do que mulheres em comportamentos e situações de risco, especialmente quando se consideram a idade e outros fatores da história de vida do indivíduo. Quais os motivos para essa diferença?

De acordo com Daly e Wilson (2001), os homens se engajam em mais atividades de risco do que as mulheres porque, durante a evolução, deve ter havido uma maior competição intragrupal por parceiras sexuais entre homens do que entre mulheres. Assim, enquanto o sucesso reprodutivo das mulheres estaria limitado pelo acesso a recursos materiais e ao dispêndio de sua própria energia para gerar e manter sua prole, para os homens, o sexo com menor investimento na prole determinaria a quantidade de parceiras sexuais como o 
fator mais importante para o seu sucesso reprodutivo. Ou seja, homens aceitariam mais riscos porque apresentam uma maior variância no sucesso reprodutivo; em contraste, entre as mulheres, um menor envolvimento em comportamentos de risco é explicável pelo fato de o sucesso reprodutivo e a sobrevivência pessoal serem fatores interdependentes (Wang e cols., 2009).

Considerando os principais achados do nosso estudo, pode-se verificar que o domínio Fertilidade e reprodução foi percebido como o mais arriscado tanto por homens quanto por mulheres. Esse resultado indica semelhanças na percepção de risco ao sucesso reprodutivo para ambos os sexos. Por outro lado, ao serem comparados com os resultados do estudo original de Wang e cols. (2009), nota-se uma convergência de achados apenas para os domínios Desafio ambiental e Acasalamento e recursos, e algumas divergências de resultados, como os relacionados à Probabilidade de engajamento no domínio Competição intergrupal. Os maiores escores médios de homens e mulheres do estudo original foram referidos à probabilidade de engajamento em comportamentos de risco relacionados à Competição intergrupal, justamente o domínio que recebeu os escores mais baixos no presente estudo.

Tratando-se de mecanismos psicológicos supostamente universais, a concordância de resultados não é surpreendente, mas a divergência requer uma reflexão adicional. Enquanto respostas precisas dependerão de novos estudos, pode-se sugerir a possibilidade de que as diferenças entre os achados indiquem interpretações relacionadas ao efeito de amostra e de particularidades ecológicas e culturais.

Uma vez que variáveis relacionadas ao ciclo vital estão envolvidas no comportamento de risco, parte da divergência dos resultados pode ser atribuída à menor média de idade dos participantes americanos, pertencente ao estudo de Wang e cols. (2009), em comparação com a amostra do presente estudo. A amostra americana encontrava-se concentrada numa faixa etária em que Competição intergrupal, Acasalamento e Fertilidades são questões dominantes. Em contraste, cerca de um quarto da amostra do nosso estudo tinha mais de 32 anos, e mais de um quinto declarou ser casado ou coabitar. Ademais, a proporção de sujeitos da amostra com filhos foi de cerca de um quarto da amostra total, em contraste com $6,9 \%$ no estudo original.
Por outro lado, práticas sociais culturalmente sancionadas, como a formação de agremiações universitárias e a intensa competitividade associada ao individualismo da sociedade dos Estados Unidos (Beekun, Stedham, \& Yamamura, 2003) podem estar na base das diferenças encontradas na Percepção, Atratividade e Propensão ao envolvimento em situações de risco. Essa diferença não é incompatível com a abordagem evolucionista, nem significa a atribuição de padrões comportamentais à cultura como uma entidade extracorpórea e independente. Tooby e Cosmides (1995) discutem o conceito de cultura e apontam para diferentes fenômenos comumente chamados de cultura, os quais, de fato, descrevem fenômenos diferentes: metacultura, cultura evocada e cultura epidemiológica. Os dois últimos conceitos de cultura, a evocada e a epidemiológica, podem estar envolvidos no fenômeno das diferenças entre populações na propensão ao risco, na medida em que os traços culturais são construídos como respostas coordenadas a condições locais, e ao mesmo tempo, difundem-se na população através de diversos mecanismos de transmissão cultural. No entanto, em qualquer caso, a cultura é sempre reconstruída pelas mentes dos indivíduos.

Nesse sentido, diferenças na percepção, atratividade e probabilidade de engajamento em situações mais ou menos arriscadas devem ser mediadas pelo conhecimento e familiaridade prévios, individual e compartilhados, que os indivíduos têm das situações avaliadas. Assim, um mecanismo universal se expressa em comportamento variável, segundo as características do ambiente em questão.

Em síntese, os achados do presente estudo respaldam a concepção de que o comportamento de risco é orientado por certas regularidades, e deve ser definido em relação a ambientes diferentes, com consequências nos resultados comportamentais. Esses resultados sugerem ainda, de modo consistente, que não é possível a compreensão do comportamento de risco sem a consideração das particularidades e similaridades comportamentais relacionadas a gênero e a efeitos de domínio analisados.

Não obstante, faz-se necessário enfatizar que o tema comportamento de risco está presente em muitos estudos em psicologia da saúde, relacionando fatores de personalidade e contextuais que implicam no envolvimento de pessoas em atividades perigosas para a saúde (Garcia \& Costa Junior, 2008), e que também 
podem ser compreendidos, assim como os achados do presente estudo, à luz da teoria evolucionista.

Pode-se fazer ainda a ressalva de algumas limitações envolvidas na presente pesquisa. $\mathrm{O}$ instrumento utilizado foi apenas ligeiramente ajustado para as condições brasileiras. Nesse sentido, Wang e cols. (2009) já tinham apontado uma crítica ao questionário por eles proposto, sugerindo que fosse adicionada uma maior quantidade de itens para cada dimensão. Desse modo, tratando-se de contextos diferentes, é possível que estudos brasileiros mais intensivos de validação sejam necessários para descrever com mais precisão o fenômeno em questão. Sugere-se que maiores contribuições poderão surgir também a partir de um estudo de amostra mais ampla de sujeitos, na qual novos aspectos relacionados à história de vida possam estar presentes.

Ainda deve-se considerar que a literatura aponta a necessidade de reflexões evolucionistas mais amplas sobre os problemas de domínios específicos e a definição de variáveis interferentes. Entretanto, as grandes mudanças nos modos de vida contemporâneos colocam desafios à teoria: quais os riscos evolutivamente relevantes hoje? Admitindo-se que eles sejam similares em substância aos riscos do ambiente evolucionista, as diversas ecologias locais teriam o potencial de afetar os padrões comportamentais envolvidos?

Portanto, pesquisas posteriores, que reforcem as condições ecológicas e culturais brasileiras, relacionando a variável gênero com outras variáveis da história de vida (como o status conjugal, parental e a idade), e com a propensão ao comportamento de risco, podem constituir importantes achados na área da perspectiva evolucionista.

\section{REFERÊNCIAS}

Barros, M. V. G., \& Nahas, M. (2001). Comportamento de risco, autoavaliação do nível de saúde e percepção de estresse entre trabalhadores da indústria. Revista de Saúde Pública, 35, 554563.

Beekun, R. I., Stedhan, I., \& Yamamura, J. H. (2003). Business ethics in Brazil and the U.S.: A comparative investigation. Journal of Business Ethics, 42, 267-279.

Colares, V., Franca, C., \& Gonzalez, E. (2009). Condutas de saúde entre universitários: Diferenças entre gêneros. Cadernos de Saúde Pública, 25, 521-528.
Courtenay-Quirk, C., Pals, S., Colfax, G., McKirnan, D., Gooden, L., \& Eroglu, D. (2008). Factors associated with sexual risk behavior among persons living with HIV: Gender and sexual identity group differences. AIDS Behavior, 12, 685-694.

Daly, M., \& Wilson, M. (2001). Risk-taking, intrasexual competition, and homicide. Nebraska Symposium on Motivation, 47, 136.

Garcia, K. S. L., \& Costa Junior, M. L. (2008). Conduta antissocial e consumo de álcool em adolescentes escolares. Revista Latino-Americana de Enfermagem, 16, 299-305.

Kruger, D. J., Wang, X. T., \& Wilke, A. (2007). Towards the development of an evolutionary valid domain-specific riskytaking scale. Evolutionary Pschology, 5, 555-568.

Lejuez, C. W., Simmons, B. L., Aklin, W. N., Daughters, S. B., \& Dvir, S. (2004). Risk- taking propensity and risky sexual behavior of individuals in residential substance use treatment. Addictive Behaviors, 29 1943-1647.

Martins, S. S, Tavares, H., Lobo, D. S. S., Galetti, A. M., \& Gentil, V. (2004). Pathological gambling, gender, and risk-taking behaviors. Addictive Behaviors, 29, 1231-1235.

Reyna, V. F., \& Rivers, S. E. (2008). Editorial: Current theories of risk and rational decision making. Developmental Review, 28, $1-11$.

Schoemaker, P. J. H. (1993). Determinants of risk-taking: Behavioral and economic views. Journal of Risk and Uncertainty, 6, 49-73.

Silveira, M. F., Béria, J. U., Horta, B. L., \& Tomasi, E. (2002). Autopercepção de vulnerabilidade às doenças sexualmente transmissíveis e Aids em mulheres. Revista de Saúde Pública, 36, 670-677.

Tooby, J., \& Cosmides, L. (1995). The psychological foundations of culture. Em J. H. Barkow, L. Cosmides \& J. Tooby (Orgs.), The adapted mind: Evolutionary psychology and the generation of culture (pp. 19-136). New York: Oxford University Press.

Trivers, R. L. (1972). Parental investment and sexual selection. Em B. Campbell (Org.), Sexual selection and the descent of man (pp. 136-179). Chicago: Aldine.

Wang, X. T., Kruger, D. J., \& Wilke, A. (2009). Life history variables and risk-taking propensity. Evolution and Human Behavior, 30, 77-84.

Weber, E. U., Blais, A. R., \& Betz, N. E. (2002). A domainspecific risk-attitude scale: Measuring risk perceptions and risk behaviors. Journal of Behavioral Decision Making, 15, 263290.

Yamamoto, M. E. (2009). Introdução: Aspectos históricos. Em E. Otta \& M. E. Yamamoto (Orgs.), Fundamentos de psicologia: Psicologia evolucionista (pp. 1-9). Rio de Janeiro: Koogan. 